\title{
CD34 Positive
}

National Cancer Institute

\section{Source}

National Cancer Institute. CD34 Positive. NCl Thesaurus. Code C148068.

An indication that CD34 expression has been detected in a sample. 